\title{
Diretrizes Curriculares na saúde e as mudanças nos modelos de saúde e de educação
}

\section{Curriculum guidelines for health and alterations in health and education models}

Carlos Otávio Fiúza Moreira', Maria Socorro de Araújo Dias²,3

'Departamento de Ciências Sociais, Escola Nacional de Saúde Pública, Fundação Oswaldo Cruz (FIOCRUZ) - Rio de Janeiro (RJ), Brasil. ${ }^{2}$ Centro de Ciências da Saúde, Curso de Graduação em Enfermagem, Universidade Estadual Vale do Acaraú (UVA) - Sobral (CE), Brasil. ${ }^{3}$ Escola de Formação em Saúde da Família Visconde de Sabóia (EFSFVS) - Sobral (CE), Brasil.

DOI: http://dx.doi.org/10.7322/abcshs.v40i3.811

\section{RESUMO}

Artigo produzido a partir de pesquisa bibliográfica e de questões formuladas para uma conferência no módulo "Educação na Saúde II" do Mestrado Profissional da Rede Nordeste de Formação em Saúde da Família. O texto discute questões dos campos da saúde e da educação relacionadas à emergência de novos modelos de saúde e de formação; analisa também as Diretrizes Curriculares Nacionais (DCN) de três cursos da área da saúde (Medicina, Enfermagem e Odontologia) como expressão desses novos modelos para saúde e para a educação. Uma questão transversal ao artigo são as relações entre os campos da saúde e da educação. Para isto, lança-se mão do conceito de campo, desenvolvido pelo sociólogo Pierre Bourdieu. Considera-se, ainda, o desafio de transformar as DCN em currículos e programas, ferramentas ou dispositivos necessários para fazer a passagem do que está prescrito para os cenários de ensino e aprendizagem.

Palavras-chave: educação; saúde; currículo.

\section{ABSTRACT}

Article produced from bibliographic research and questions formulated for a conference in the "Education in Health II" module in the Northeastern Network of Family Health Training, Professional Master's program. The paper discusses questions from health and education fields related to the emergence of new health and training models; it analyses the National Curriculum Guidelines (NCG) from three courses in the health area (Medical School, Nursing and Dentistry) as expression of these new models for health and education. A crosscutting issue to the article is the relationship between health and education fields. For this purpose, we resorted to the field concept, developed by the sociologist Pierre Bourdieu. It is still considered the challenge of transforming the NCG in curriculums and programs, tools or devices necessary to transfer what is written to the teaching and learning scenarios.

Keywords: education; health; curriculum. 


\section{INTRODUÇÃO}

Na obra de Bourdieu ${ }^{1}$, campo é definido como um microcosmo do espaço social, com regras próprias, agentes legítimos que neles atuam e autonomia relativa em relação aos outros campos. Cada campo é um recorte do espaço social. Isto significa que os campos estão relacionados, exercem forças entre si, mas desfrutam também de certa autonomia: nem tudo que vale para um campo tem igual reconhecimento em outro. $\mathrm{O}$ espaço social onde vivemos seria então o conjunto desses microcosmos, os campos e suas relações. As diferenças entre os campos são frutos de processo histórico, que produzem tensões e a referida autonomia relativa ${ }^{1-3}$.

Ao usarmos a expressão "campo da saúde", estamos nos referindo a um espaço de ação, de práticas, de lutas, de produção de saberes e de exercício de poder. Já a expressão "área da saúde" diz respeito a uma divisão técnica e administrativa, presente nas instituições, nos documentos oficiais, nos processos de classificação, certificação e avaliação. Essa observação expressa o esforço de compreensão e uso desses termos nesta discussão sobre as Diretrizes Curriculares Nacionais (DCN) e as mudanças nos modelos de saúde e de educação.

\section{Contexto de emergência das Diretrizes Curriculares Nacionais: os campos da Saúde e da Educação}

A Constituição de 1988 é marco histórico para o Brasil, especialmente para o campo da saúde, na medida em que instituiu um novo sistema de saúde. A criação do Sistema Único de Saúde (SUS) indicava uma mudança considerável no modo de conceber e produzir saúde, considerando-a como "direito de todos e dever do Estado"4. Sua institucionalização sinalizava para transformações no modo de atuar no campo da saúde.

Era inevitável que esse modelo passasse a influir na formação dos profissionais de saúde. A outorga constitucional do SUS para "ordenar a formação na área da saúde" não cancela a incumbência legal do Ministério da Educação (MEC) de regular a formação no país. É uma questão instalada nas fronteiras políticas e jurídicas entre dois campos. Como indica Machado ${ }^{5}$, o uso de determinados termos "sugere a importância do envolvimento da autoridade sanitária em decisões relevantes para a saúde, mesmo as que estejam sob a responsabilidade direta de outros setores". Ressalta-se que o diálogo institucional entre o Ministério da Saúde (MS) e outros setores do Estado não estão isentos de controvérsias e disputas.

Voltemos a 1988. Estávamos distante da promulgação das DCN para os cursos da saúde. As primeiras foram lançadas em 2001. Enquanto o sistema de saúde estava sendo reordenado para um novo modelo, a formação profissional para atuar nesse sistema continuava orientada pela lógica do antigo modelo.

Essa premissa nos ajuda a entender a emergência e os sentidos das DCN, já que não estamos apenas narrando histórias/ ordenando ideias, mas atualizando a escrita e a interpretação de processos, tendo em vista as questões presentes. Em texto de 2001 sobre o Programa de Saúde da Família (PSF), Paim ${ }^{6}$ lembra que seu processo de implantação valorizou certos componentes estruturais do sistema de saúde. Contudo, a questão do cuidado, inscrita nos modelos de atenção, foi secundarizada, como política de governo. Isso reforça a necessidade de alinhamento da formação para o cuidado requerido.

A discussão sobre a regulação da formação dos profissionais de saúde estivera presente de diversas formas no Brasil desde 1930 mas ganhou força na década de 1990, chegando às DCN. Se revisitarmos as Conferências Nacionais de Saúde, veremos que temas relacionados a essa aparecem de forma desigual em seus relatórios ${ }^{7}$. A hipótese aqui apresentada é a de que as DCN dos cursos da saúde estão relacionadas de forma explícita a mudanças do modelo de atenção à saúde. Após a promulgação das DCN, políticas públicas específicas à formação em saúde e relacionadas ao novo modelo começaram a ser implantadas. A partir desse contexto, a formação em saúde deixou de ser um tema marginal no MS.

Não foi ao acaso que as discussões sobre a formação tenham se dado relacionadas ao debate do novo modelo de saúde. Era evidente a inadequação do perfil profissional. Para Silva e $\operatorname{Trad}^{9}$, a institucionalização do Programa Saúde da Família (PSF) demonstrou isso ao buscar efetivar a Atenção Primária à Saúde (APS), levando-o a ser considerado como uma política governamental estratégica ${ }^{10}$. Para Germano et al. ${ }^{11}$, a Estratégia Saúde da Família (ESF) busca mudar "[...] paradigmas cristalizados, incorporando um novo olhar, um novo pensar e um novo fazer, no qual o foco passe a ser a saúde e não a doença; a família e não o indivíduo; a equipe e não o médico [...]".

O exposto indica a necessária aproximação do perfil de competências dos profissionais de saúde das necessidades dos processos de trabalho ${ }^{12}$. Profissionais formados no modelo biomédico têm dificuldades para atuar no novo contexto ${ }^{13}$.

As DCN, que emergiram nesse cenário, deveriam funcionar como parâmetros para as mudanças curriculares, na perspectiva de formar profissionais para o novo modelo ${ }^{14}$. Dessa perspectiva, as DCN podem ser lidas como sínteses programáticas possíveis à formação de profissionais, que incorporassem aspectos estruturais de um sistema de saúde legalmente implantado há mais de uma década, mas que recebia e, a nosso ver, ainda recebe profissionais quase avessos aos seus princípios.

Para que as DCN dos cursos da saúde fossem escritas e promulgadas, diversas iniciativas de agentes do campo da saúde contribuíram. Uma instituição importante nesse debate foi a Rede Unida, enquanto expressão com fusão de vários movimentos a exemplo do projeto Integração Docente-assistencial (IDA). No qual:

Personagens importantes da reforma sanitária brasileira estiveram participando [...], naquele momento cumpria o papel fundamental de romper o isolamento de projetos que continuavam esparsos no cenário nacional, ainda no contexto da ditadura militar ${ }^{15}$.

O IDA foi relevante para o fortalecimento do SUS, mas com baixo impacto na formação. Neste, "os serviços eram considerados mais como cenários que como parceiros"15. 
Outro destaque foi o projeto "Uma Nova Iniciativa na formação de profissionais de saúde" (UNI), parceria entre universidade, serviços de saúde e organizações comunitárias orientada pela cogestão do processo de trabalho colaborativo e compartilhamento de poderes, saberes e recurso ${ }^{16}$.

A Rede Unida se configurou como um ator político e institucional de articulação frente às discussões e proposições sobre a formação em saúde. Uma das constatações da época foi que não havia, nos espaços do SUS, tematização adequada sobre a formação em saúde. Como indicamos, uma hipótese para se compreender essa demora em trazer a formação para o primeiro plano era o predomínio da visão de que "as mudanças na organização do sistema de saúde antecederiam e determinariam as mudanças na formação profissional"15. Trata-se de um dilema político ainda pertinente.

Uma questão levada à frente por essas iniciativas foi o fortalecimento de espaços de discussão que articulavam as instâncias de definição das políticas públicas de saúde e de educação: o MS e o MEC, os Conselhos Nacionais de Saúde, de Educação, de Secretários de Saúde e de Secretarias Municipais de Saúde, etc.

Ainda no campo da saúde, é importante destacar que foi constituído um discurso relativamente consistente e propositivo sobre a necessidade de mudanças na formação. Considerando que o SUS deveria ordenar a formação de profissionais da saúde, outros atores institucionais do campo, como as associações de ensino médico e de enfermagem e a diretoria executiva nacional de estudantes também pressionaram órgãos como o Conselho Nacional de Saúde e a Coordenação de Recursos Humanos do MS para que se manifestassem no debate, apresentando sugestões para a elaboração dos perfis dos profissionais, tendo como referência o sistema de saúde ${ }^{15}$.

Algumas considerações sobre o contexto institucional que viabilizou as DCN no âmbito do MEC podem nos ajudar a compreender certas características das diretrizes. Lembremos da mencionada autonomia relativa dos campos, de que fala Bourdieu. No caso em análise, damos como exemplo as diferenças institucional e programática entre dois campos. Apesar da crescente aproximação entre o MS e o MEC, em anos recentes, no que diz respeito a processos de formação em saúde, a distinção permanece e é relevante também para pensarmos sobre os desafios da intersetorialidade.

Se no campo da saúde a implantação do SUS foi um marco histórico e balizador das políticas, a Lei de Diretrizes e Bases da Educação (LDB 9.394/96) serviu como referência para reorientação do sistema educacional brasileiro. Se a LDB não expressa um projeto de sociedade tão abrangente como o da Reforma Sanitária, ela sintetiza um acordo possível entre os atores sociais envolvidos em sua elaboração.

A ideia das DCN está presente de forma clara no Artigo 53, inciso II, dessa lei geral:

No exercício de sua autonomia, são asseguradas às universidades, sem prejuízo de outras as seguintes atribuições: II. Fixar os currículos dos seus cursos e programas, observadas as diretrizes gerais pertinentes ${ }^{17}$.
Um dos objetivos das DCN era tornar os currículos mais flexíveis, eliminando os currículos mínimos, com seu elevado grau de detalhamento de disciplinas e cargas horárias. As Instituições de Ensino Superior (IES) tinham então baixa margem de liberdade para produzir e implantar seus próprios projetos pedagógicos, concretizados nos currículos. As observações do então gestor do Departamento de Política do Ensino Superior do MEC, Rubens Martins, dão algumas pistas neste sentido.

No contexto atual, onde há rápidas mudanças no conhecimento, é no mecanismo de educação continuada que está a chave para que o ensino superior acompanhe tais transformações. E este aspecto dinâmico só é viável dentro de uma estrutura como a das diretrizes curriculares, que vão possibilitar às IES definirem diferentes perfis de seus egressos e adaptarem estes perfis a estas transformações [...]. É o contrário do currículo mínimo, que acaba por definir cursos estáticos e perfis profissionais também estáticos ${ }^{18}$.

Assim, uma perspectiva mais dinâmica para a montagem dos currículos se tornava possível, na medida em que era facultado às IES a construção de diferentes percursos de formação. Foi nesse contexto institucional que o MEC convocou as IES a enviar propostas para a elaboração das DCN, ampliando a discussão para além do âmbito dos membros das Comissões de Especialistas de Ensino de cada área. Houve consultas e audiências públicas.

Segundo o relator das DCN para os cursos da área da saúde, o professor Efrem Maranhão, entre os vários documentos consultados para a elaboração dessas diretrizes, estavam a Constituição Federal de 1988, a LDB, o Relatório Final da $11^{\text {a }}$ Conferência Nacional de Saúde, Documentos da Organização Pan-americana de Saúde, da Organização Mundial de Saúde, da Rede Unida, a Declaração Mundial sobre Educação Superior no Século XXI da UNESCO, além de editais do MEC e instrumentos que regulamentam o exercício das profissões da saúde. As propostas finais de cada curso foram encaminhadas pela Secretaria de Educação Superior do MEC ao Conselho Nacional de Educação, que as homologou ${ }^{19}$.

Como mostramos, no campo da saúde, uma série de atores se mobilizaram, articulando discussões e elaborando propostas sobre a formação profissional dos trabalhadores da saúde, pois se reconhecia que, apesar do processo de implantação e consolidação do SUS em curso, a formação ainda permanecia uma área crítica. Assim, a partir do ano 2000, outro paradigma de formação, tendo como referência um novo modelo de atenção à saúde, foi inscrito nas DCN dos cursos da área da saúde. É sobre isso que falaremos agora.

\section{As Diretrizes Curriculares Nacionais como expressão de um novo modelo para a formação em saúde}

Para ampliar a discussão sobre os sentidos do novo paradigma de formação que orienta as DCN dos cursos da saúde, tomamos como referência as diretrizes dos cursos de Enfermagem, 
Medicina e Odontologia. A escolha teve como base o seguinte critério: são as três profissões com formação universitária que compõem a equipe de referência da ESF. Apresentamos a seguir uma leitura tópica das DCN dos cursos indicados.

Os textos das DCN de todos os cursos da saúde têm basicamente a mesma estrutura:

1. perfil do egresso/profissional;

2. competências e habilidades;

3. conteúdos curriculares;

4. estágios e atividades complementares;

5. organização do curso;

6. acompanhamento e avaliação.

Percebe-se também que, além de formatos e redações semelhantes, há uma parte comum a todas as profissões referente às competências gerais. Há também partes específicas de cada profissão. A análise que elaboramos guarda semelhanças com a apresentada por Maranhão ${ }^{19}$, mas com distintas referências aos textos das DCN. O objetivo é destacar algumas características do profissional a ser graduado nos cursos da área da saúde, tendo em vista um novo modelo de formação e de cuidado em saúde.

A análise dessas características pode ser associada a um determinado perfil profissional para atuação no campo da saúde, que por sua vez está relacionado a um modelo de atenção à saúde. Mesmo em algumas partes que se referem às competências específicas de cada profissão, é possível destacar características esperadas dos egressos que se distinguem do chamado modelo biomédico, centrado nas doenças dos indivíduos e com ênfase na especialização profissional.

Em contraste com o referido modelo e a título de exemplo, vejamos o Art. $3^{\circ}$ das DCN da Medicina. Ali se indica que o perfil do egresso é o profissional médico

com formação generalista, humanista, crítica e reflexiva, capacitado a atuar, pautado em princípios éticos, no processo de saúde-doença em seus diferentes níveis de atenção, com ações de promoção, prevenção, recuperação e reabilitação à saúde, na perspectiva da integralidade da assistência, com senso de responsabilidade social e compromisso com a cidadania, como promotor da saúde integral do ser humano ${ }^{20}$.

Esse propósito de formar um profissional generalista, com visão de mundo humanista, crítica e reflexiva, é uma diretriz geral, válida para todos os cursos da saúde, que se relaciona ao conceito ampliado de saúde e a uma mudança de perspectiva do sistema de saúde.

Entre as competências gerais (Art. $4^{\circ}$ ) para a formação dos profissionais, podemos destacar como parte de uma nova perspectiva de atenção à saúde a de "estar aptos a desenvolver ações de prevenção, promoção, proteção e reabilitação da saúde, tanto em nível individual quanto coletivo" ${ }^{20}$. Parece claro que o foco da formação se desloca da doença para a saúde. Onde havia basicamente o indivíduo como referência das ações, aparece também o coletivo, a saúde coletiva. Além das clássicas ações de reabilitação e prevenção, a promoção da saúde ganha espaço, ampliando os sentidos das ações de saúde.

Outro destaque, ainda no artigo ( $\left.4^{\circ}\right)$, que sinaliza para a formação relacionada a outro modelo de atenção é o trabalho em equipe multiprofissional. Nas DCN para a Medicina, a atuação em equipes multiprofissionais aparece nas competências específicas, no Art. $5^{\circ}$, alínea XXI, onde se indica a necessidade do médico "atuar em equipe multiprofissional"; o tema do trabalho multiprofissional reaparece nos conteúdos especiais. No caso das DCN de Enfermagem, esse tema é tratado na alínea XXII do artigo $5^{\circ}$, com a redação: "integrar as ações de enfermagem às ações multiprofissionais" ${ }^{21}$. As sutis diferenças nas redações dos textos não desfazem a clara aposta no trabalho em equipes multiprofissionais.

Digno de comentário também é o Art. $6^{\circ}$, que trata dos conteúdos essenciais dos cursos de graduação. Junto aos conhecimentos biológicos clássicos, há a indicação da necessidade de "compreensão dos determinantes sociais, culturais, comportamentais, psicológicos, ecológicos, éticos e legais, nos níveis individual e coletivo, do processo saúde-doença" ${ }^{20,21}$. Se a questão dos determinantes sociais hoje pode parecer algo do senso comum no campo da saúde, não é nada óbvia a sua compreensão para quem é formado em currículo estruturado e focado apenas nos fenômenos biológicos referentes ao corpo dos indivíduos. É importante notar que nas DCN para os cursos da saúde não se aludem apenas ao termo "determinantes sociais". Amplia com referências aos fatores "culturais, comportamentais, psicológicos, ecológicos, éticos e legais" que estruturam o processo saúde-doença. Isso significa o aumento de exigências para as instituições de formação na área da saúde, posto que o cumprimento de tal diretriz suponha a inclusão de novos componentes curriculares que possam dar conta de tais conteúdos, algo não muito simples para o corpo docente tradicional dos cursos de graduação das profissões da saúde. Isso pode requerer, por exemplo, a incorporação de docentes com formação em outras áreas de saber.

Relacionada a um novo paradigma de educação é a perspectiva de uma formação permanente, expressa nas competências gerais de todos os cursos, indicando ser algo comum a todas as profissões da saúde. Trata-se da ideia de que os profissionais de saúde devem "aprender a aprender" e ter compromisso com a sua educação e das futuras gerações de profissionais.

Aqui estão sinalizações claras de mudanças que só começaram a ser efetivas em políticas e programas lançados após a criação da Secretaria de Gestão do Trabalho e da Educação na Saúde (SGTES) no MS, em 2003. Essa secretaria assumiu a responsabilidade de formular políticas orientadoras da gestão, formação, qualificação e regulação dos trabalhadores da saúde no Brasil ${ }^{7,8}$.

Com um contexto favorável às mudanças no campo da saúde, estas não tardaram a aparecer em forma de políticas e programas do MS. Foram lançados desde então a Política Nacional de Educação Permanente e programas em que o SUS aparece como locus de formação, como VER-SUS, Promed, Pró-Saúde, Pet, Provab, Mais Médicos, GraduaSus, além do incentivo às residências. 
Importante destacar as estratégias educacionais propostas nas DCN. No caso da Medicina, no Art. 12, a alínea II indica

utilizar metodologias que privilegiem a participação ativa do aluno na construção do conhecimento e a integração entre os conteúdos, além de estimular a interação entre o ensino, a pesquisa e a extensão/assistência ${ }^{20}$.

Já nas DCN de Enfermagem, esse tema é tratado no Art. 14, e a alínea VII indica que se deve assegurar "o estímulo às dinâmicas de trabalho em grupos, por favorecerem a discussão coletiva e as relações interpessoais" ${ }^{21}$. Esses enfoques distintos podem ser relacionados a diferentes estratégias metodológicas empregadas com relativa frequência em cursos de Medicina e Enfermagem, como Aprendizagem Baseada em Problemas (ABP) e a problematização, respectivamente. As DCN para Odontologia indicam, além da utilização de metodologias ativas, a participação ativa dos alunos, a integração dos conhecimentos das ciências básicas com os das ciências clínicas e a instituição de programas de iniciação científica como método de aprendizagem ${ }^{22}$.

A participação ativa dos alunos na construção do conhecimento, o estímulo às dinâmicas de trabalho em grupo e a integração entre os conhecimentos das ciências básicas e os das ciências aplicadas à clínica requerem mudanças consideráveis na estrutura curricular e nos processos de ensino e aprendizagem, indicando a necessidade de apropriação consistente, por parte dos professores e preceptores, de metodologias adequadas. Enfim, para desenvolver currículos orientados pelas DCN, outras competências são esperadas do corpo docente. Esse desafio foi percebido pelos gestores do MS, logo após a promulgação das DCN.

Neste sentido, uma das primeiras iniciativas da SGTES foi um curso de especialização em ativação de processos de mudança na formação superior de profissionais de saúde. Esse curso objetivou formar profissionais capazes de desencadear um pensamento crítico e ações estratégicas com vistas a dinamizar mudanças na formação superior em saúde. Entre os temas, estava o uso de metodologias ativas de ensino e aprendizagem ${ }^{23}$.

O último tema que recortamos das DCN é a ampliação dos cenários de aprendizagem. Como discutido, é importante pensar sobre efeitos possíveis das DCN na formação e na prática dos futuros profissionais de saúde, considerando que tais efeitos passam necessariamente pela configuração dos currículos da graduação. Nesses, ocupa lugar de destaque a diversificação dos cenários de aprendizagens.

Nas DCN do curso de Medicina, encontra-se no Art. 12 a indicação de "utilizar diferentes cenários de ensino-aprendizagem permitindo ao aluno conhecer e vivenciar situações variadas de vida, da organização da prática e do trabalho em equipes multiprofissionais" (alínea VI). Indica ainda que se deve "vincular, através da integração ensino-serviço, a formação médico-acadêmica às necessidades sociais da saúde, com ênfase no SUS" (alínea VIII).

Essa é uma questão importante e sua execução não é simples. Em discussão sobre o tema e a diversificação de cenários de ensino e trabalho, Laura Feuerwerker, Heloniza Costa e Ligia Rangel lembravam que "cenários de aprendizagem" se referem não somente aos locais onde se realizam as práticas, mas aos sujeitos envolvidos, aos conteúdos das práticas, aos métodos didáticos utilizados, à utilização de tecnologias, aos conhecimentos e habilidades previstos. Inclui ainda os preceitos éticos e os processos de trabalho. As autoras ressaltam a complexidade do conceito e, ao mesmo tempo, a potência da estratégia "para induzir mudanças mais profundas no processo de formação profissional e como elemento em si mesmo constitutivo de nova maneira de pensar a formação profissional"24.

Essas considerações parecem muito produtivas para esta discussão, pois uma visão restrita de "diferentes cenários de aprendizagem" pode associar a expressão apenas a espaços físicos distintos das salas de aula e que comportariam a transposição de práticas escolares para além dos muros das escolas. Contudo, para se efetivar a referida proposição das DCN de Medicina - "utilizar diferentes cenários de ensino-aprendizagem permitindo ao aluno conhecer e vivenciar situações variadas de vida, da organização da prática e do trabalho em equipes multiprofissionais" (Art. 12, alínea VI) - é preciso compreender tais cenários como espaços de práticas, locais de trabalho e de produção de serviços de saúde, com organizações e dinâmicas próprias; é preciso também construir os mecanismos de cooperação entre as instâncias ensino e os serviços. Enfim, o objetivo dessa diretriz é tornar possível a vivência dos futuros profissionais de saúde em espaços reais de prática, e isto inclui a "incorporação de estudantes e docentes ao processo de produção de serviços" ${ }^{2}$.

Além das referências aos princípios e diretrizes do SUS, que nos remetem para um novo modelo de saúde, as DCN dos cursos estão desenhadas com uma perspectiva de desenvolvimento de competências, modelo de ensino e aprendizagem bem distinto daquele que tem orientado, de uma maneira geral, a formação universitária no Brasil. As DCN indicam que a aprendizagem deve ter como foco a resolução de problemas e a evidência científica, o que possibilitaria a articulação entre teoria e prática; indicam também que os processos saúde-doença devem ser relacionados aos aspectos biológicos, sócio-econômicos, culturais e psicológicos; e que o aluno deve ser considerado como o sujeito do processo de aprendizagem ${ }^{25,26}$.

O cumprimento efetivo dessas diretrizes implica em uma radical mudança da graduação. Não por acaso, mais de uma década depois da promulgação das DCN algumas IES ainda trabalham com currículos distantes daqueles previstos nas DCN. Ou seja, os desafios para operar com os efeitos curriculares dessas diretrizes parecem contínuos.

\section{CONCLUSÃO}

Tanto a legislação como as políticas de Estado e de Governo precisam de instâncias de mediação para se efetivar. Isso não é diferente com a aplicação das DCN dos cursos da saúde. Neste sentido, elas devem se transformar em currículos e programas dos cursos de graduação. Esses são dispositivos capazes de realizar a 
passagem do que está previsto nas diretrizes para os processos de formação nos cenários de ensino e aprendizagem.

Isto significa também a translação das regras gerais, nacionais, para instâncias locais com relativa autonomia: as IES com suas culturas e histórias próprias. Nesse âmbito, a construção dos currículos segue uma lógica quase artesanal, que demanda participação coletiva, constituição de comissões, disputas de propostas, interpretações da legislação, acordos, aplicação das decisões tomadas. Além das referências às $\mathrm{DCN}$, currículos e programas são também avaliados indiretamente pelos seus efeitos educacionais. Isso se dá por meio dos mecanismos de avaliação do ensino superior regulados pelo MEC. Fecha-se, com isso, um ciclo móvel da estrutura de ensino superior na área da saúde, no qual se inserem instituições da área da educação: do SUS às DCN, das DCN aos cursos de graduação, dos cursos aos alunos. Transformados em profissionais, eles ingressam no trabalho com as competências desenvolvidas nos processos de formação.

\section{REFERÊNCIAS}

1. Bourdieu P. O poder simbólico. Rio de Janeiro: Bertrand Brasil; 1989

2. Bourdieu P. Razões práticas: sobre a teoria da ação. Campinas: Papirus; 2008.

3. Bourdieu P. Escritos de educação. Petrópolis: Vozes; 1998

4. Brasil. Presidência da República. Constituição da República Federativa do Brasil de 1988. Brasília: 1988

5. Machado CV. Direito universal, política nacional: o papel do Ministério da Saúde na política de saúde brasileira de 1990 a 2002. Tese (Doutorado) - Instituto de Medicina Social da Universidade do Estado do Rio de Janeiro, Rio de Janeiro, 2005.

6. Paim JS. Saúde da Família: espaço de reflexão e de contra-hegemonia. Interface. 2001;5(9):143-6. http://dx.doi.org/10.1590/S1414-32832001000200010

7. Pereira IDF. A formação profissional em saúde no Brasil pós-1988: aspectos das Conferências Nacionais de Saúde e das Políticas Públicas. Dissertação (Mestrado) - Escola Nacional de Saúde Pública, Rio de Janeiro, 2013.

8. Rezende M. A articulação educação-saúde (AES) no processo de formulação das políticas nacionais voltadas a formação de nível superior dos profissionais de saúde. Tese (Doutorado) - Escola Nacional de Saúde Pública, Rio de Janeiro, 2013.

9. Silva IZQJ, Trad LAB. O trabalho em equipe no PSF: investigando a articulação técnica e a interação entre os profissionais. Interface. 2005;9(16):25-38.

http://dx.doi.org/10.1590/S1414-32832005000100003

10. Brasil. Ministério da Saúde. Secretaria de Assistência à Saúde. Coordenação de Saúde da Comunidade. Saúde da Família: uma estratégia para a reorientação do modelo assistencial. Brasília: Ministério da Saúde; 1997.

11. Germano RM, Formiga JMM, Bezerra de Melo MN, Vilar RLA Almeida Junior JJ. Capacitação das equipes do PSF: desvendando uma realidade. In: Castro JL. Gestão do trabalho no SUS: entre o visível e o oculto. Natal: Observatório RH-NESC/UFRN; 2007. p. 105-32.

12. Barrêto ICHC, Oliveira EM, Andrade LOM, Martins Junior T, Osawa M, Vicente A, et al. A Residência em Saúde da Família em Sobral: um ano formando especialistas em larga escala. Sanare: Rev Políticas Públicas. 2000;2(3):18-28.

13. Ceccim RB, Feuerwerker LCM. O quadrilátero da formação para a área da saúde: ensino, gestão, atenção e controle social. Physis. 2004;14(1):41-65.

http://dx.doi.org/10.1590/S0103-73312004000100004
14. Brasil. Ministério da Saúde. A educação permanente entra na roda: pólos de educação permanente em saúde: conceitos e caminhos a percorrer. 2 ed. Brasília: Ministério da Saúde; 2005.

15. Feuerwerker LCM, Costa HOG, Barbosa N, Khalil ME. O processo de construção e de trabalho da Rede Unida. Divulg Saúde Debate. 2000;22:9-17.

16. Feuerwerker LCM. Algumas reflexões sobre o desenvolvimento do Programa UNI. Divulg Saúde Debate. 2000;22:63-70.

17. Brasil. Lei $n^{\circ}$ 9.394, de 20 de dezembro de 1996. Estabelece as diretrizes e bases da educação nacional. Brasília; 1996.

18. Martins RO. Entrevista: É hora de estabelecer as Diretrizes Curriculares. Rev Olho Mágico. 2004:4(16).

19. Maranhão E. A construção coletiva das Diretrizes Curriculares Nacionais dos Cursos de Graduação da Saúde: uma contribuição para o Sistema Único de Saúde. In: Almeida MJ. Diretrizes Curriculares Nacionais para os cursos universitários da área de saúde. Londrina: Rede Unida; 2003.

20. Rossoni E, Lampert J. Formação de profissionais para o Sistema Único de Saúde e as diretrizes curriculares. Bol Saúde. 2004;18(1):87-98.

21. Brasil. Resolução do Conselho Nacional de Educação e da Câmara de Educação Superior $n^{\circ}$ 4, de 7 de novembro de 2001. Institui Diretrizes Curriculares Nacionais do Curso de Graduação em Medicina. Disponível em: http://portal.mec.gov.br/cne/arquivos/ pdf/CES04.pdf. Acesso em: 06 ago. 2013.

22. Brasil. Resolução do Conselho Nacional de Educação e da Câmara de Educação Superior no 3, de 7 de novembro de 2001. Institui Diretrizes Curriculares Nacionais do Curso de Graduação em Enfermagem. Disponível em: http://portal.mec.gov.br/cne/ arquivos/pdf/CES03.pdf. Acesso em: 06 ago. 2013.

23. Brasil. Resolução do Conselho Nacional de Educação e da Câmara de Educação Superior 3, de 19 de fevereiro de 2002. Disponível em: http://portal.mec.gov.br/cne/arquivos/pdf/CES032002.pdf. Acesso em: 06 ago. 2013.

24. Parente JRF. Preceptoria e tutoria na residência multiprofissional em saúde da família. Sanare. 2008;7(2):47-53.

25. Fundação Oswaldo Cruz. Educação à Distância. Curso de Especialização em ativação de processos de mudança na formação superior de profissionais de saúde. Cad Especializando. Rio de Janeiro: Fiocruz; 2005.

26. Feuerwerker L, Costa H, Rangel ML. Diversificação de cenários de ensino e trabalho sobre necessidades/problemas da comunidade. Divulg Saúde Debate. 2000;22:36-48. 